\title{
HYDROFLUORIC ACID-BASED ULTRASONIC UPGRADING OF OIL SHALE AND ITS STRUCTURE CHARACTERIZATION
}

\author{
WEINA SONG, YONGLI DONG*, LIMEI XUE, \\ HUIXIAN DING, ZHE LI, GUOJIANG ZHOU
}

Modern Analysis and Research Center

Heilongjiang Institute of Science and Technology

Harbin 150027, China

\begin{abstract}
The effect of hydrofluoric acid (HFA) on the upgrading and structure of oil shale from Yilan county, Heilongjiang province, China was investigated by the ultrasonic treatment of the shale in a mild water bath. The oil shale samples were characterized by X-ray diffraction (XRD), X-ray fluorescence (XRF) and scanning electron (SE) microscopies, as well as Fourier transform infrared (FTIR) spectroscopy, and densimetry. The results of the study indicate that the HFA-based ultrasonic treatment exhibited a better performance in upgrading oil shale than the ordinary mechanical stirring method. It was also found that the concentration of HFA significantly influenced upgrading performance and the separation degree of inorganic minerals. The kerogen content in oil shale increased with increasing concentration of HFA. The total content of $C, H, O$ and $N$ in oil shale reached 98.2\% when $40 \%$ HFA was used. Oil shale is composed mainly of a chainlike cyclic saturated organic material called kerogen, and inorganic minerals such as quartz, kaolinite, illite, siderite and pyrite. These materials integrate into a smooth lithified sandwich by the hydrogen bond and intermolecular forces. Via the ultrasonic treatment under mild conditions, kaolinite and siderite can be removed from oil shale with the use of a weak HFA, while a stronger HFA is needed to leach illite and quartz. Then kerogen with a loose porous structure is obtained.
\end{abstract}

Keywords: oil shale, ultrasonic treatment, kerogen, inorganic mineral, hydrofluoric acid.

\section{Introduction}

Oil shale (OS) is an unconventional resource of oil and gas. After carbonization, it can release shale oil, which is similar in composition to crude oil.

\footnotetext{
* Corresponding author: e-mail yonglidong@yahoo.cn
} 
Therefore, shale oil is also called "synthetic oil". The world oil shale reserves are considerable. Converted into shale oil, these are estimated to be 400 billion tons, which is 5.4 times more than those of obtainable crude oil all over the world. Hence, oil shale is considered a promising substitute for crude oil [1,2]. Due to the rapid development of global industry and economy in the 21 st century, the demand for oil has significantly increased lately. Political, economic and also military activities aiming at scrambling for crude oil resources are disquieting. Therefore it is most desirable that a new oil shale conversion technology for obtaining oil should be developed.

Oil shale is a fossil fuel that leaves much ash after combustion, is of low calorific value and contains a lot of inorganic minerals, which impart the shale low oil-yielding rate and low quality [3-6]. The organic kerogen cannot be separated from oil shale via conventional gravity or froth floatation [7]. Today, chemical scientists are mostly dealing with analysis of the structure and composition of the organic constituent and inorganic minerals present in oil shale, as well as the molecular structure of kerogen and lithification characteristics of inorganic minerals. Oil shale is also examined for the effect of different acids and alkali on its demineralization, pyrolysis kinetics and oil-yielding rate, etc. [8-11]. To avoid the formation of acid waste, extensive research is carried out to control the $\mathrm{pH}$ by using a $\mathrm{NaOH}$ solution, resulting in the formation of several precipitates, e.g., $\mathrm{Al}(\mathrm{OH})_{3}$. Thereafter these precipitates are easily converted to chemical compounds, e.g., $\gamma-\mathrm{Al}_{2} \mathrm{O}_{3}[12,13]$. The separation of kerogen and minerals from oil shale has several disadvantages, involving the formation of various acid wastes, being time consuming and necessitating the use of harsh experimental conditions (e.g., heating to boiling) [11, 14]. Therefore, the development of a straightforward and green method for separation of kerogen and minerals from oil shale is highly desirable.

In this paper, we developed a novel ultrasonic method for separation of kerogen and minerals from oil shale with the use of HFA. The oil shale samples were upgraded by using the ultrasonic treatment in mild conditions. The upgraded samples were further characterized by XRD, XRF and SE microscopies, as well as FTIR spectroscopy, and densimetry. The influence of the concentration of HFA on the upgrading and structure of Yilan oil shale was investigated.

\section{Experimental}

\subsection{Materials}

Raw oil shale samples were obtained from the Yilan open-pit, Heilongjiang province, northeastern China. Hydrofluoric acid (HFA, 40\%), supplied by Sinopharm Chemical Co., Ltd (China), was of analytical grade. Superpure water (resistance value $18.25 \mathrm{M} \Omega \cdot \mathrm{cm}$ ) was prepared using a PINE-TREE XYB-60-H Water Purifier in this work. 


\subsection{Upgrading experiment}

The upgrading experiments with oil shale samples were carried out using a KQ-100DE Ultrasonic Cleaner (Kunshan Ultrasonic Instrument Co., Ltd, China), a ZM-D-F Vibrating Mill (Changchun Keguang Mechanics and Electronics Co., Ltd, China), a DZ-2BC Vacuum Oven (Tjanjin Taisite Instrument Co., Ltd, China), a Sartorius BT224S Electronic Balance (Sartorius Instrument Co., Ltd, China), and a TDL-40B Centrifuge (Hunan Xingke Instrument Co., Ltd, China), to treat the samples in each step of the experiment.

The oil shale samples were crushed and then ground in a vibrating mill to the required particle size. The samples were sieved to obtain a fraction size of $0.2 \mathrm{~mm}$, and dried at $50{ }^{\circ} \mathrm{C}$ under vacuum. $10 \mathrm{~g}$ of oil shale sample was added to $40 \mathrm{~mL}$ of $5 \%, 10 \%, 20 \%, 30 \%$ and $40 \%$ HFA solution, and then the slurry was subjected to ultrasonic vibration for $5 \mathrm{~h}$ in a water bath at $60{ }^{\circ} \mathrm{C}$. The mixture was filtered to obtain a dark solid and then washed with superpure water until the filtrate became neutral. Finally the dark solid was dried under vacuum. The samples treated with a $5 \%, 10 \%, 20 \%, 30 \%$ or $40 \%$ HFA solution were named OS-5F, OS- $10 \mathrm{~F}$, OS-20F, OS-30F and OS-40F, respectively. A reference sample treated with $40 \%$ HFA without using ultrasound was prepared under similar conditions by stirring, and was named OS-40F-NU.

\subsection{Characterization}

X-ray diffraction measurements were carried out on a Bruker D8 Advance $\mathrm{X}$-ray diffractometer, Germany, with $\mathrm{Cu} \mathrm{K} \alpha_{1}(\lambda=0.15406 \mathrm{~nm})$ radiation $(40 \mathrm{kV}, 40 \mathrm{~mA})$. A Perkin Elmer Spectrum 100 FT-IR spectrometer was used to measure the wavelengths of functional groups of different OS samples, using a $\mathrm{KBr}$ pellet, the scan range was from 4000 to $370 \mathrm{~cm}^{-1}$. SEM measurements were run on a CamScan MX2600FE scanning electron microscope, UK, operating at $20 \mathrm{kV}$. The elements content of samples was measured on a Bruker S4 Explorer X-ray fluorescence spectrometer, Germany. An MDMDY 350 automatic density analyzer (Zhongshan Meidi Analytical Instrument Co., China) was employed to measure the density of OS samples.

\section{Results and discussion}

\subsection{Component analysis}

$\mathrm{X}$-ray fluorescence spectrometry was used to identify the components of raw and upgraded OS samples. The results are shown in Table 1. In raw OS samples, the major elements were Si (11.76\%), Al (5.166\%), Fe (0.9272\%), $\mathrm{S}(0.3120 \%)$ and $\mathrm{K}(0.1800 \%)$, trace elements being $\mathrm{Ca}, \mathrm{Ti}, \mathrm{Mg}, \mathrm{Mn}, \mathrm{Zn}$ and $\mathrm{Sr}$. The results indicate that the Yilan oil shale is of a high Si content. With the HFA concentration increasing from 5 to $30 \%$, the total content of $\mathrm{C}, \mathrm{H}$, $\mathrm{O}$ and $\mathrm{N}$ significantly increased, from 81.3 to $97.4 \%$. With a further increase 
Table 1. XRF analysis of oil shale samples

\begin{tabular}{|l|l|l|l|l|l|l|l|}
\hline \multirow{2}{*}{ Element } & \multicolumn{7}{|c|}{ Content in samples, \% } \\
\cline { 2 - 8 } & \multicolumn{1}{|c|}{ OS } & OS-5F & OS-10F & OS-20F & OS-30F & OS-40F & OS-40F-NU \\
\hline $\mathrm{C}, \mathrm{H}, \mathrm{O}, \mathrm{N}$ total & 81.3 & 84.1 & 86.8 & 95.4 & 97.4 & 98.2 & 96.8 \\
$\mathrm{Si}$ & 11.76 & 11.25 & 11.10 & 2.01 & 0.13 & 0.13 & 0.18 \\
$\mathrm{Al}$ & 5.166 & 2.526 & 0.936 & 0.990 & 0.861 & 0.196 & 0.846 \\
$\mathrm{Fe}$ & 0.9272 & 0.4073 & 0.2764 & 0.2537 & 0.3838 & 0.2316 & 0.5833 \\
$\mathrm{~S}$ & 0.312 & 0.3410 & 0.4480 & 0.6640 & 0.7273 & 0.8298 & 0.8911 \\
$\mathrm{~K}$ & 0.1800 & 0.1780 & 0.1580 & 0.1327 & 0.0531 & 0.0115 & 0.1070 \\
$\mathrm{Ca}$ & 0.1163 & & & & & & \\
$\mathrm{Ti}$ & 0.0998 & & & & & & \\
$\mathrm{Mg}$ & 0.0766 & & & & & & \\
$\mathrm{Mn}$ & 0.0041 & & & & & & \\
$\mathrm{Zn}$ & 0.0023 & & & & & & \\
$\mathrm{Sr}$ & 0.0045 & & & & & & \\
\hline
\end{tabular}

in the HFA concentration to $40 \%$ this value reached $98.2 \%$. So, the upgrading capability of oil shale attained maximum with the use of a $40 \%$ HFA solution. The reference sample OS-40F-NU, which was treated with $40 \%$ HFA without using ultrasound, was also investigated. Its total content of $\mathrm{C}$, $\mathrm{H}, \mathrm{O}$ and $\mathrm{N}$ was only $96.8 \%$ vs $98.2 \%$ and $97.4 \%$ in OS-40F and OS-30F, respectively.

Compared with the raw OS sample, the Si content of OS-10F reduced only by $0.66 \%$ and this value decreased sharply, by $10.97 \%$, after the HFA concentration increased to $30 \%$. With increasing HFA concentration, the Al content of samples gradually decreased from 5.166 (OS) to $0.936 \%$ (OS-10F), showing only a slight variation in OS-20F and OS-30F samples. The Al content of OS- $40 \mathrm{~F}$ decreased to $0.196 \%$. The content of $\mathrm{K}$ diminished slowly in samples treated with HFA of a concentration lower than $20 \%$, but decreased abruptly, from 0.1327 to $0.0115 \%$, when the HFA concentration grew from 20 to $40 \%$. The Fe content showed a clear decrease, from 0.9272 in OS to $0.2764 \%$ in OS-10F, when $10 \%$ HFA was used, but decreased insignificantly with increasing HFA concentration even up to $40 \%$. While those mineral components were successfully eliminated in varying degrees, the $\mathrm{S}$ content of upgraded samples exhibited an upward trend with increasing HFA concentration.

The XRF results demonstrate that the ultrasonic method exhibits a better performance in the upgrading of oil shale in mild conditions than the ordinary mechanical stirring. The total content of $\mathrm{C}, \mathrm{H}, \mathrm{O}$ and $\mathrm{N}$ may reach $98.2 \%$ when $40 \%$ HFA is used. At the HFA concentration as low as 5 and $10 \%$, demineralization of Al- and Fe-containing inorganic minerals takes place. When a stronger HFA is used, Si can be almost fully leached by $30 \%$ HFA. However, the leaching of other Al- and K-containing minerals requires a relatively strong HFA. Nevertheless, not all iron minerals present in oil shale can be totally removed by HFA, but only a few. Furthermore, the sulfide contained in oil shale cannot be removed by HFA neither, and the 
increase of S content should be attributed to the decrease of total mineral content and aggregation of sulfide, leading to the increase of a relative content of $\mathrm{S}$ in the samples.

\subsection{XRD results}

XRD measurements were carried out to investigate the crystalline phase composition of raw and upgraded oil shale samples (Fig. 1). It can be seen that the OS sample is composed mainly of quartz $\left(\mathrm{SiO}_{2}\right)$, kaolinite $\left[\mathrm{Al}_{2}\left(\mathrm{SiO}_{5}\right)(\mathrm{OH})_{4}\right]$, siderite $\left(\mathrm{FeCO}_{3}\right)$ and illite $\left[\mathrm{K}_{4} \mathrm{Al}_{4} \mathrm{Si}_{7} \mathrm{AlO}_{20} \cdot \mathrm{H}_{2} \mathrm{O}\right][10,11$, 15]. As demonstrated by the XRD patterns of OS-5F and OS- $10 \mathrm{~F}$, the diffraction peaks of siderite disappear in weakly acidic conditions. The intensity of diffraction peaks of kaolinite also decreases until the peaks disappear in OS-20F. With increasing HFA concentration, the intensity of diffraction peaks of quartz decreases in OS-20F, disappearing in $\mathrm{OS}-30 \mathrm{~F}$ and OS-40F. The diffraction peaks of illite, which are present in OS to OS-20F, are not observed in the OS-30F sample. Nevertheless, after the elimination of abundant crystalline minerals, several weak peaks of pyrite $\left(\mathrm{FeS}_{2}\right)$ can be observed in OS-20F, OS-30F and OS-40F, which reveals that some iron minerals failed to be removed by HFA and the aggregation of sulfide occurs to a certain extent [4]. Consequently, the diffraction peaks of inorganic minerals in OS-40F can no longer be detected, except trance pyrite. Moreover, the pattern of OS-30F shows a small broad peak at around $20^{\circ}$ which can be attributed to amorphous organic carbon and hydrogen compounds. This peak is more clearly observed in OS-40F.

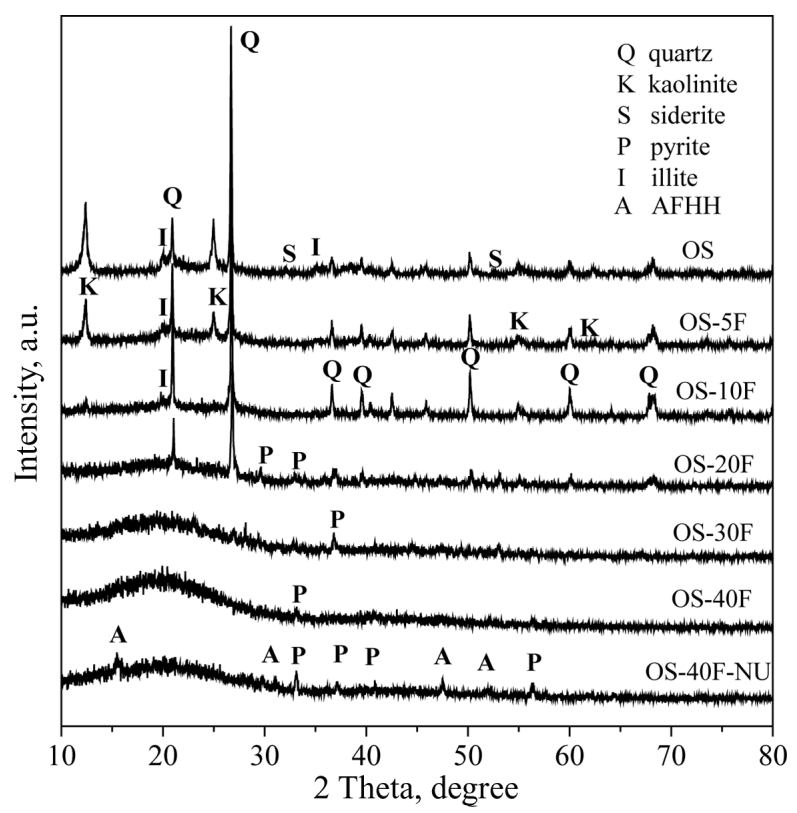

Fig. 1. XRD patterns of oil shale samples. 
In the reference sample OS-40F-NU some diffraction peaks are attributable to pyrite $\left(\mathrm{FeS}_{2}\right)$, while several are ascribed to aluminium fluoride hydroxide hydrate (AFHH). AFHH may be formed as an intermediate in the reaction of HFA with Al-containing minerals. These results are in good agreement with the XRF analysis data revealing that the total content of Al, $\mathrm{Fe}, \mathrm{S}, \mathrm{Si}$, and $\mathrm{K}$ in OS-40F-NU is higher than that in OS-30F and OS-40F.

While interpreting XRD and XRF results, it can be seen that a remarkable decrease of the content of $\mathrm{Al}$ and $\mathrm{Fe}$ in oil shale samples is observed while the Si content displays no clear change in OS-5F and OS-10F samples. This indicates that inorganic minerals kaolinite and siderite in oil shale can be decomposed in weakly acidic conditions, but the $\mathrm{Si}$ present in kaolinite cannot be fully eliminated. With increasing HFA concentration, the reaction of $\mathrm{Si}$ with HFA is accelerated when $20 \%$ HFA is used. As a result, almost $99 \%$ of the Si present in quartz and other minerals is removed from OS-30F and OS-40F samples. The decrease of $\mathrm{K}$ and $\mathrm{Al}$ content of these samples can be attributed to the elimination of illite by a relatively strong HFA. Then, after the HFA-based demineralization, the amorphous organic mixture of hydrocarbon compounds ( $98.2 \%$ by wt), i.e. kerogen, is obtained in the OS-40F sample. The ultrasonic method used for upgrading of oil shale reveals a better performance than the ordinary mechanical stirring, while the concentration of HFA significantly influences the process.

\subsection{FTIR spectroscopic analysis}

FTIR spectrometry was used to investigate the organic and inorganic functional groups of untreated and upgraded oil shale samples. As can be seen in Fig. 2, the inorganic mineral part of OS consists mainly of kaolinite (3699, 3621, 3660, 1033, 1008 and $\left.913 \mathrm{~cm}^{-1}\right)$ and quartz $(1096,799,694$ and $\left.469 \mathrm{~cm}^{-1}\right)$. The shoulder peak at $3380 \mathrm{~cm}^{-1}$ is attributable to the hydrogen bond formed during the lithification of minerals with kerogen in oil shale. After treatment with HFA of varying concentrations, the kaolinite content in OS-10F considerably decreases, and most quartz can be removed by $30 \%$ HFA. This leads to the appearance of a faint peak of $\mathrm{FeS}_{2}$ at $625 \mathrm{~cm}^{-1}$ in OS-30F. Furthermore, a gradual disappearance of the shoulder peak at 3380 $\mathrm{cm}^{-1}$ is indicative of that the hydrogen bonds in oil shale are broken. When the HFA concentration reaches $40 \%$, the FTIR spectra of OS-30F mainly display the characteristic peaks of kerogen and obvious peak shifts are obtained, unlike OS. The peaks at 2935, 2851 and $1453 \mathrm{~cm}^{-1}$ are characteristic of aliphatic $-\mathrm{CH}_{2}-$ and $-\mathrm{CH}_{3}$, indicating that kerogen is composed mainly of chainlike and cyclic saturated materials, while a broad peak at $1276 \mathrm{~cm}^{-1}$ gives evidence of the existence of a symmetrical $-\mathrm{CH}_{3}$. The peak at $1622 \mathrm{~cm}^{-1}$ corresponds to the $\mathrm{C}=\mathrm{C}$ stretching band of the aromatic group $[10,16,17]$.

These results are in accordance with XRF and XRD data on demineralization. After the upgrading of oil shale, the organic components of kerogen have well been preserved, which indicates that the energy of ultrasound cannot 


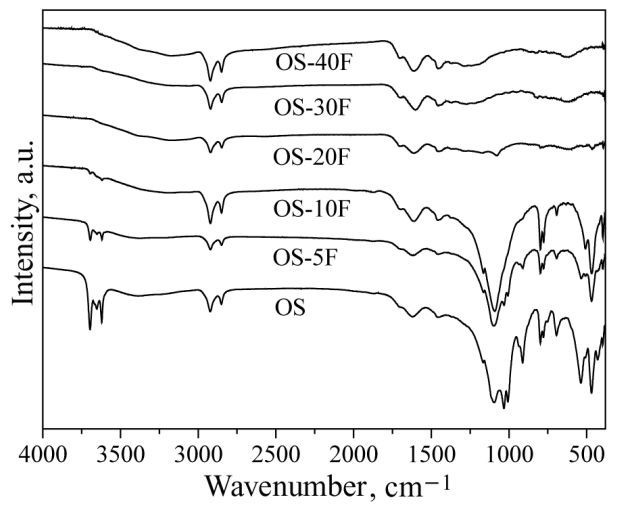

Fig. 2. FTIR spectra of oil shale samples.

break the chemical bonds $\mathrm{C}-\mathrm{C}$ and/or $\mathrm{C}-\mathrm{O}$ in kerogen and it just weakens or opens the intermolecular hydrogen bonds and van der Waals forces between inorganic minerals and kerogen in oil shale [18]. This demonstrates that the ultrasonic decomposition method used in mild conditions enables an effective removal of inorganic minerals from the high-Si oil shale and retains kerogen with an intact structure.

\subsection{Density analysis}

The density of raw oil shale sample is $2.067 \mathrm{~g} / \mathrm{cm}^{3}$ as shown in Fig. 3. The density of samples decreases with increasing HFA concentration. The density of OS-20F decreases from $2.067 \mathrm{~g} / \mathrm{cm}^{3}$ in OS to $1.486 \mathrm{~g} / \mathrm{cm}^{3}$ when the HFA concentration increased to $20 \%$. The ultrasonic treatment leads to the leaching of high-density materials from oil shale, which makes its structure incompact and density-depressing. However, with increasing HFA concentration, especially from 30 to $40 \%$, the density of samples only slightly decreases from 1.385 to $1.316 \mathrm{~g} / \mathrm{cm}^{3}$, which may be associated with a small variation in the component composition of OS-30F and OS-40F samples as shown by XRF, XRD and FTIR techniques.

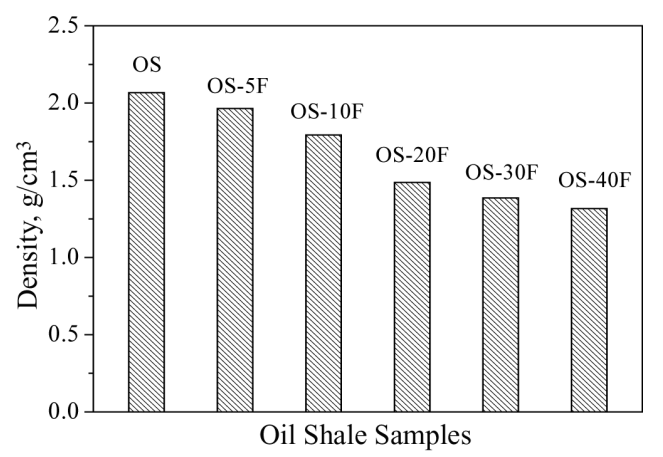

Fig. 3. The density of oil shale samples treated with HFA of different concentrations. 


\subsection{SEM analysis}

Scanning electron microscopic (SEM) images of oil shale samples are presented in Fig. 4. It can clearly be seen that the raw oil shale sample
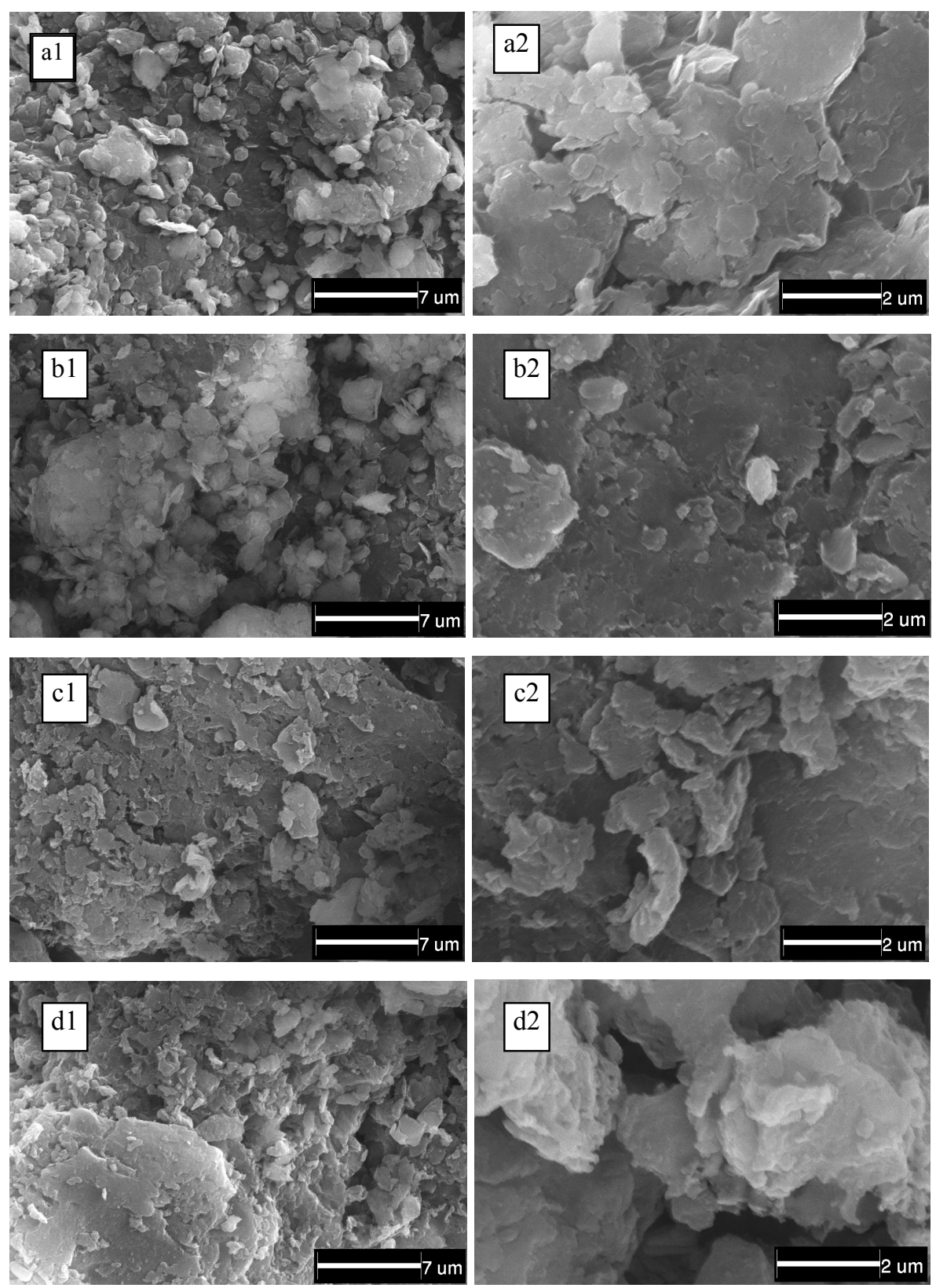

Fig. 4. SEM images of OS (a1, a2), OS-5F (b1, b2), OS-20F (c1, c2) and OS-40F (d1, d2). 
possesses a compact lithified smooth-edged sandwich structure (Fig. 4, a1 and a2). After the HFA-based ultrasonic treatment of samples, some hollows and crevices are formed on the lithified sandwich surface. With increasing HFA concentration, the smooth edges in the sandwich become asymmetric and even disappear owing to the elimination of most lithofacies minerals. At last, these hollows and crevices in the sandwich transform to pores and it assumes a porous structure (Fig. 4, c1 and c2). Fig. $4 \mathrm{~d} 1$ and d2 illustrate that, after leaching of most inorganic minerals, pure kerogen is of a loose porous structure and contains organic macromolecules. This further confirms the results of density analysis of upgraded oil shale samples.

\section{Conclusions}

(1) The HFA-based ultrasonic treatment of oil shale shows a better upgrading performance than the ordinary mechanical stirring, while the concentration of the acid significantly influences the process.

(2) After the ultrasonic treatment with $40 \% \mathrm{HFA}$ at $60{ }^{\circ} \mathrm{C}$ for $5 \mathrm{~h}$, the total content of $\mathrm{C}, \mathrm{H}, \mathrm{O}$ and $\mathrm{N}$ of the upgraded $\mathrm{OS}$ sample may reach $98.2 \%$.

(3) The Yilan oil shale is composed mainly of a chainlike cyclic saturated organic material kerogen, and some inorganic minerals, such as quartz, kaolinite, illite, siderite, pyrite. Kerogen and minerals integrate into a smooth lithified sandwich.

(4) Through the ultrasonic treatment, with the employment of HFA of increasing concentrations, different crystalline minerals are gradually eliminated from the compact sandwich of oil shale. Siderite and kaolinite are eliminated by a weak HFA, while the removal of quartz and illite necessitates the use of a stronger HFA. As a result, kerogen with a loose porous structure is obtained.

\section{Acknowledgment}

We are grateful for the support from the Natural Science Foundation of China (No. 21203058) and the Youth Science Foundation of Heilongjiang Province of China (No. QC2010050).

\section{REFERENCES}

1. Chen, H. J., Liu, Z. J., Zhu, J. W., Fu, Z. R. Economic evaluation of oil shale utilization. Geology and Resources, 2011, 20, 50-55 (in Chinese). 
2. Liu, Z. J., Liu, R. Oil shale resource state and evaluating system. Earth Science Frontiers, 2005, 12, 315-323 (in Chinese).

3. Xu, Y. M., Qi, J., Shi, J. W., He, D. M., Wang, D. M., Zhang, Q. M. Technique of preparing modified silica from oil shale residue. Oil Shale, 2011, 28(2), 309320.

4. Wang, D. M., Xu, Y. M., He, D. M., Guan, J., Zhang, Q. M. Investigation of mineral composition of oil shale. Asia-Pac. J. Chem. Eng., 2009, 4(5), 691-697.

5. Yan, J. W., Jiang, X. M., Han, X. X. Study on the characteristics of the oil shale and shale char mixture pyrolysis. Energ. Fuel., 2009, 23(12), 5792-5797.

6. Patterson, J. H. A review of the effects of minerals in processing of Australian oil shales. Fuel, 1994, 73(3), 321-327.

7. Al-Otoom, A. Y. An investigation into beneficiation of Jordanian El-Lajjun oil shale by froth flotation, Oil Shale, 2008, 25(2), 247-253.

8. Karabakan, A., Yürüm, Y. Effect of the mineral matrix in the reactions of shales. Part 2. Oxidation reactions of Turkish Göynük and US western reference oil shales. Fuel, 2000, 79(7), 785-792.

9. Ballice, L. Stepwise chemical demineralization of Göynük (Turkey) oil shale and pyrolysis of demineralization products. Ind. Eng. Chem. Res., 2006, 45(3), 906-912.

10. Rose, H. R., Smith, D. R. An investigation of thermal transformations of the products of oil shale demineralization using infrared emission spectroscopy. Energ. Fuel., 1993, 7(2). P. 319-325.

11. Al-Harahsheh, A., Al-Harahsheh, M., Al-Otoom, A., Allawzi, M. Effect of demineralization El-lajjun Jordanian oil shale on oil yield. Fuel Process. Technol., 2009, 90(6), 818-824.

12. Li, Y., Feng, Z. Y., Xue, X. X., He, Y., Qiao, G. B. Ecological utilization of oil shale by preparing silica and alumina. Journal of Chemical Industry and Engineering, 2008, 59, 1051-1057 (in Chinese).

13. Feng, Z. Y., Li, Y., Yang, H., Xue, X. X. Study on preparation of hydrated silica from oil shale and application of dispersants. Mining and Metallurgy, 2008, 17, 43-47 (in Chinese).

14. Ballice, L. Effect of demineralization on yield and composition of the volatile products evolved from temperature-programmed pyrolysis of Beypazari (Turkey) oil shale. Fuel Process. Technol., 2005, 86(6), 673-690.

15. Heistand, R. N., Humphries, H. B. Direct determination of organic carbon in oil shale. Anal. Chem., 1976, 48(8), 1192-1194.

16. Ballice, L., Yüksel, M., Saglam, M., Schulz, H., Hanoglu, C. Application of infrared spectroscopy to the classification of kerogen types and the thermogravimetrically derived pyrolysis kinetics of oil shales. Fuel, 1995, 74(11), 1618-1623.

17. Tissot, B. P., Vandenbroucke, M. Geochemistry and pyrolysis of oil shales. In: Geochemistry and Chemistry of Oil Shales. ACS Symposium Series, 230 (F. P. Miknis, J. F. McKay, eds.). American Chemical Society, Washington, D. C., 1983, 1-11.

18. Wang, N., Sun, C. G., Li, B. Q. Review of the study on low molecular weight compounds of coal. Coal Conversion, 1997, 20, 19-23 (in Chinese).

Presented by J. Kann

Received March 17, 2012 